\title{
Boldine Attenuates Cancer Cell Growth in an Experimental Model of Glioma In vivo
}

Daniéli Gerhardt ${ }^{*}$, Gabriela Bertola ${ }^{1}$, Andressa Bernardi ${ }^{1}$, Eliza Nicoloso Simões Pires ${ }^{1}$, Rudimar Luiz Frozza ${ }^{1}$, Maria Isabel A Edelweiss ${ }^{2}$, Ana Maria O Battastini ${ }^{1}$ and Christianne G Salbego ${ }^{1}$

${ }^{1}$ Departamento de Bioquímica, Instituto de Ciências Básicas da Saúde, Universidade Federal do Rio Grande do Sul (UFRGS), Porto Alegre, RS, Brasil ${ }^{2}$ Departamento de Patologia, Hospital de Clínicas de Porto Alegre, Universidade Federal do Rio Grande do Sul (UFRGS), Porto Alegre, RS, Brasil

\begin{abstract}
Plants are important sources of biologically active natural products which differ in terms of structure and biological properties. One of the biological activities of plant compounds that attracts great interest is the ability to exert anticancer activity in different cancer types. Boldine is a natural alkaloid that occurs abundantly in the leaves and bark of Peumus boldus. It has been demonstrated to present anticancer properties in bladder carcinoma and in glioma cancer cells in vitro. In the present study, we evaluated the effect of boldine in an in vivo experimental model of glioma and investigated some parameters of toxicity in healthy animals. Our results show that boldine did not cause any toxicity in the animals treated. In rats with implanted glioma and treated with boldine a significant reduction in tumor size was observed. Pathological analysis of implanted gliomas showed a reduction in the mitotic index as well as in other histological characteristics in boldine treated animals, indicating a less invasive and proliferative tumor. No change was observed in the VEGF expression in treated animals. Our results suggest that boldine could be a promise adjuvant therapy in the treatment of malignant gliomas.
\end{abstract}

\section{Keywords: Boldine; Toxicity; Glioma; Antitumor}

\section{Introduction}

Plants are important sources of biologically active natural products which differ in terms of structure and biological properties. Some of the isolated plant constituents such as flavonoids, alkaloids, and others are responsible for many biological activities, such as antiinflammatory activity, analgesic activity, antiallergenic activity, amog others. One of the biological activities of plant compounds that has attracted great interest is the ability to exert anticancer activity in different types of cancer. Boldine, (S)-2,9-dihydroxy-1,10-dimethoxy-aporphine, is an alkaloid that occurs abundantly in the leaves and bark of boldo (Peumus boldus Mol.) a widely distributed native tree of Chile [1,2]. Boldo infusions have been traditionally employed in folk medicine and used as a medicinal plant for the treatment of digestive and hepatobiliary disorders [2-4]. Furthermore, it has been recognized as an herbal remedy in several pharmacopeias in South America and Europe [2]. Chemically pure boldine has been shown to exhibit, amongst others, immuno-modulating [5], smooth muscle relaxing [6], antipyretic and antiinflammatory $[7,8]$ and neuroleptic-like [9] properties.

However, in recent years, boldine has attracted more attention in relation to its potent antioxidative and cytoprotective properties [10-12]. Low micromolar concentrations of boldine have been shown to prevent both enzymatic and non-enzymatic mediated damage to biological systems. In vitro, boldine inhibits the free-radical-mediated initiation and propagation of the peroxidative damage induced to various membrane types such as liver homogenates, hepatic microsomes and erythrocytes, and it blocks the free radical-dependent lysis of red blood cells and intact hepatocytes $[10,11,13]$. These boldine cytoprotective actions are associated with its potent antioxidative activity, once this molecule acts as an efficient hydroxyl radical scavenger in biological systems [11]. Apart from its antioxidant activity, boldine has also been demonstrated to have anticancer properties against bladder carcinoma and glioma cancer cells $[14,15]$.

Malignant gliomas are the most common primary tumors in the human brain. Current treatment includes surgical resection, radiation therapy, and chemotherapy. However these neoplasms are extremely resistant and frequent tumor recurrence results in poor prognosis with a mean survival time of $12-15$ months for grade IV glioma [16]. Exploring new adjuvant therapies are, therefore, necessary for improving the outcome of glioma treatment.

In our early study, we demonstrated the ability of boldine in inhibit the in vitro growth of malignant brain cells (U-138MG and $\mathrm{U}-87 \mathrm{MG}$ human glioma, and C6 rat glioma). With regard to in vivo approaches, it is not know until now whether boldine is safe to use and whether it is really effective in treating glioma grown in an orthotopic microenvironment. Once this alkaloid already has demonstrated anticancer activity in vitro, we examine the effect of this compound in an orthotopic and immunocompetent rat model of glioma that faithfully mirrors the hallmarks of human gliomas and evaluate the in vivo toxicity of boldine.

\section{Materials and Methods}

\section{Chemicals}

Cell culture media and Fetal Bovine Serum (FBS) were obtained from Gibco-Invitrogen (Grand Island, NY, USA). Boldine and Propidium iodide (PI) were obtained from Sigma Chemical Co (St. Louis, MO, USA). All other reagents were purchased from Sigma Chemical Co. (St. Louis, MO, USA) or Merck (Darmstadt, Germany). All other chemicals and solvents used were of analytical or pharmaceutical grade.

${ }^{*}$ Corresponding author: Daniéli Gerhardt, Departamento de Bioquímica, Instituto de Ciências Básicas da Saúde, UFRGS Rua Ramiro Barcelos, 2600 - anexo, CEP 90035-003, Porto Alegre, RS, Brasil, Tel: +55 (51) 3308.5547; Fax: +55 (51) 3308.5535; E-mail: danieli83@yahoo.com.br

Received April 17, 2013; Accepted May 15, 2013; Published May 17, 2013

Citation: Gerhardt D, Bertola G, Bernardi A, Simões Pires EN, Frozza RL, et al. (2013) Boldine Attenuates Cancer Cell Growth in an Experimental Model of Glioma In vivo. J Cancer Sci Ther 5: 194-199. doi:10.4172/1948-5956.1000206

Copyright: (c) Gerhardt D, et al. This is an open-access article distributed unde the terms of the Creative Commons Attribution License, which permits unrestricted use, distribution, and reproduction in any medium, provided the original author and source are credited. 


\section{Animal maintenance}

Healthy male Wistar rats (180-240 g) were obtained from inhouse breeding colonies at the "Departamento de Bioquímica", "Universidade Federal do Rio Grande do Sul" (UFRGS - Porto Alegre, Brazil). Animals were housed in cages under optimum light conditions (12:12 h light-dark cycle), temperature $\left(22 \pm 1^{\circ} \mathrm{C}\right)$, and humidity (50 to $60 \%)$, with food and water provided ad libitum. All procedures used in the present study followed the "Principles of Laboratory Animal Care" from NIH publication No. 85-23 and were approved by the local Ethics Committee on the Use of Animals. All efforts were made to minimize the number of animals and their suffering.

\section{Toxicity evalution}

Healthy animals were separated into three groups (4 animals per group) as follows: untreated (control group); treated with drugvehicle (50\% ethanol/water); treated with $50 \mathrm{mg} / \mathrm{Kg} /$ day of boldine solubilized in vehicle. These animals were treated intraperitoneally for 14 days and weighed every day. After that, animals were euthanized for toxicological experiments evaluation. Blood samples were collected by cardiac puncture. Blood smears were prepared from freshly blood, stained with May-Grünwald-Giemsa Staining and then a manual White Blood Cells (WBC) differential was performed (100 WBCs were found, counted, and categorized according to type). For enzymatic assays (alkaline phosphatase, gamma-glutamyl transpherase, alanine aminotranspherase (ALT), aspartate aminotranspherase (AST), blood samples were centrifuged at $2,500 \times \mathrm{g}$ at $4^{\circ} \mathrm{C}$ for $10 \mathrm{~min}$. The blood serum was collected and frozen at $-70^{\circ} \mathrm{C}$ until the assays were performed. The enzymes activities, which were used as markers of hepatic tissue toxicity, were measured according to the recommendations of the kit supplier (Labtest, MG, Brazil). Brain, liver, stomach, kidney, heart and lung were removed, weighted, sectioned and fixed with $10 \%$ paraformaldehyde for posterior pathological analysis. Paraffin embedded sections were stained with hematoxylin and eosin (H\&E) and analyzed under a microscope by a pathologist. For gastrointestinal tolerance study, the stomach and the intestine (duodenum, jejunum and ileum) were excised and slit open opposite to the attached mesenteric tissue. The organs were washed with saline and the mucosal surfaces were macroscopically examined for the presence or absence of hemorrhagic points and ulcers.

\section{Cell culture}

The C6 rat glioma cell line was obtained from the American Type Culture Collection (Rockville, Maryland, Md., USA). Cells were grown and maintained in Dulbecco's modified Eagle's medium supplemented with $5 \%(\mathrm{v} / \mathrm{v})$ fetal bovine serum, $2.5 \mathrm{mg} / \mathrm{mL}$ Fungizone and 100 U/L gentamicin (Schering do Brasil, São Paulo, SP, Brazil) and kept at a temperature of $37^{\circ} \mathrm{C}$, a minimum relative humidity of $95 \%$ and an atmosphere of $5 \% \mathrm{CO} 2$ in air.

\section{Glioma implantation}

Rat C6 glioma cells at around 80\% confluency were trypsinized, washed once in DMEM/5\% FBS, spun down and suspended in the same medium. A total of one million cells in a volume of $3 \mu$ l were injected at a depth of $6.0 \mathrm{~mm}$ in the right striatum (coordinates with regard to bregma: $0.5 \mathrm{~mm}$ posterior and $3.0 \mathrm{~mm}$ lateral) of anesthetized male Wistar rats [17].

\section{Treatment of animals}

After ten days of glioma implantation, the animals were randomly separated into three groups as follows: (1) untreated (control group-6 animals); (2) treated with drug-vehicle (50\% ethanol/water-6 animals); (3) treated with $50 \mathrm{mg} / \mathrm{Kg} /$ day of boldine solubilized in vehicle (8 animals). Drug and vehicle were administered intraperitoneally (i.p.) to the animals for 10 consecutive days. At the end of the treatment, the rats were decapitated and the entire brain was removed, sectioned and fixed with $10 \%$ paraformaldehyde.

\section{Pathological analysis and tumor volume quantification}

Hematoxylin and eosin (H\&E) sections (paraffin embedded) of each tumor were analyzed by a pathologist, blinded for the experimental data. For tumor size quantification, images were captured using a digital camera connected to a microscope (NikonEclipse TE300) and analyzed using Image Tool SoftwareTM. The total tumor volume $\left(\mathrm{mm}^{3}\right)$ was computed by the multiplication of the slice sections and by summing the segmented areas [18].

\section{Immunohistochemical staining}

Paraffin embedded, $5 \mu \mathrm{M}$ formalin fixed tissue sections were mounted on microscope slides. Tissue sections were then dried overnight at $60^{\circ} \mathrm{C}$, dewaxed in xylene and rehydrated with distilled water. Endogenous peroxidase was inhibited by $5 \% \mathrm{H} 2 \mathrm{O} 2$ in methanol for $15 \mathrm{~min}$. Incubation with the following antibodies was performed overnight at $4^{\circ} \mathrm{C}$ temperature: anti-Ki67 (1:200) (Dako, USA) and antiVEGF (1:30) (Dako, USA), followed by incubation with secondary antibody and Streptavidin-Avidin-Biotin (Kit Lsab, Dako, USA). The peroxidase reaction was performed using 3,3'diaminobenzidine tetrahydrochloride (DAB), according to the manufacturer's specifications. Finally, sections were counterstained with Harris haematoxylin. Glioma cell proliferation was assessed by counting the percentage of Ki67 positive glioma cell nuclei in five independent highmagnification $(\times 400)$ fields per animal. Sections of rat spleen were used as positive controls.

\section{Statistical analysis}

Data are expressed as means $\pm \mathrm{SD}$. The statistical significance among groups was assessed by one-way Analysis of Variance (ANOVA) followed by Tukey's test. Differences between mean values were considered significant when $\mathrm{p}<0.05$.

\section{Results}

\section{Macroscopic and histopathological analysis of healthy animals}

The treatment with $50 \mathrm{mg} / \mathrm{Kg} /$ day i.p. of boldine or vehicle for 14 days did not cause mortality of healthy animals. Moreover, In the period of 14 days of treatment, animals of control group and vehicle group did not improve significantly their body weights, and the treatment with boldine did not cause significant loose of body weight in rats, neither improvement (Figure 1A). Macroscopic investigation of brain, liver, kidney, lungs and hearts did not reveal any disturbance between groups. Organs weights did not present any alteration (Figure 1B). Microscopic investigation of the same organs by H\&E analysis demonstrated absence of tissue toxicity in boldine-treated rats. Representative histologies of the tissues of control and boldine-treated rats are shown in Figure 2A. Macroscopic investigation of stomach and intestine to evaluate a possible gastrointestinal damage reveals absence of hemorrhage points or ulcer in both groups (Representative pieces of intestine are seen in Figure 2B). 
Citation: Gerhardt D, Bertola G, Bernardi A, Simões Pires EN, Frozza RL, et al. (2013) Boldine Attenuates Cancer Cell Growth in an Experimental Model of Glioma In vivo. J Cancer Sci Ther 5: 194-199. doi:10.4172/1948-5956.1000206

\section{Hematological analysis}

To evaluate possible interference of boldine in blood cell populations, we prepared blood smears stained with May-GrünwaldGiemsa staining. Analyzing blood samples microscopically, we found a tendency to increase the percentage of the population of neutrophils in the detriment of the other populations (lymphocytes, monocytes) in the animals treated with boldine (Table 1).

\section{Enzymatic analysis}

Boldine treatment for 14 days did not exert hepatic toxicity in healthy animals as evaluated from determination of gamma-glutamiltranspherase and ALT (liver damage marker), AST (liver and heart unspecific damage marker), alkaline phosphatase (pancreas, liver and bone unspecific damage markers) (Figure 3).

\section{Tumor size reduction induced by treatment with boldine in} vivo

To determine whether boldine can suppress tumor growth in vivo, adult Wistar rats were inoculated in the right striatum with C6 glioma cells and, after 10 days of tumor development, they were treated with boldine $50 \mathrm{mg} / \mathrm{Kg} /$ day. After 10 -day of initiate treatment, (20 days after implantation of tumor cells) boldine group rats showed significantly lower tumor volumes compared with control and vehicle groups. There was no statistical difference between vehicle group and control group. The results demonstrated that boldine was effective to inhibit tumor growth in rat C6 glioma implantation model. Tumor volume quantification and representative images of tumors in different experimental groups are shown in Figure 4.

\section{Histopathological analysis of tumors}

Haematoxylin and eosin (H\&E) stained tumor sections examination

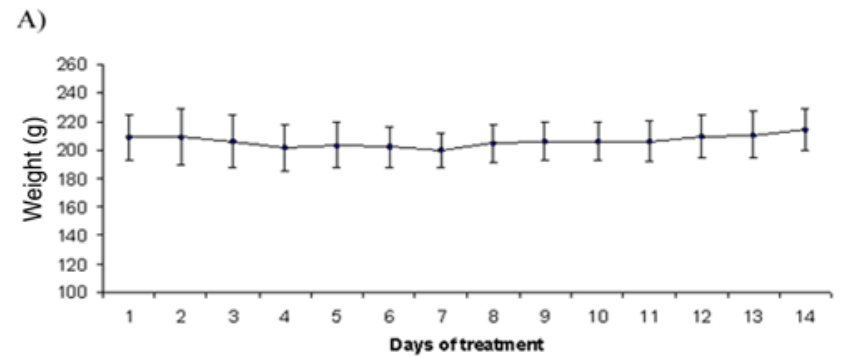

B)

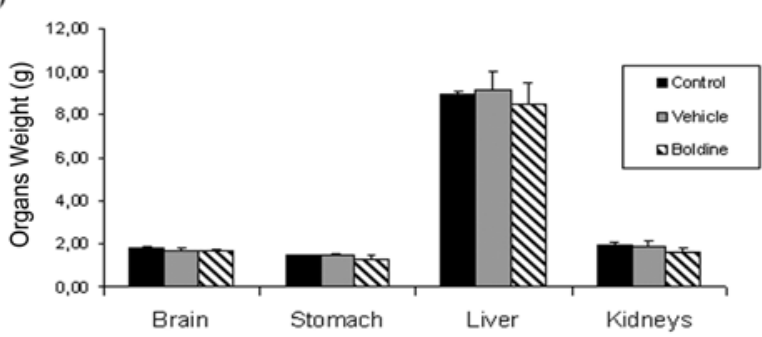

Figure 1: Body and organ weights of healthy animals treated with boldine. Animals were treated, as described in Section 2. (A) Body weights of boldinetreated healthy rats were observed daily. (B) Organs weights were observed at the end of treatment. The values are represented as means \pm S.D. of all animals used $(n=4)$.

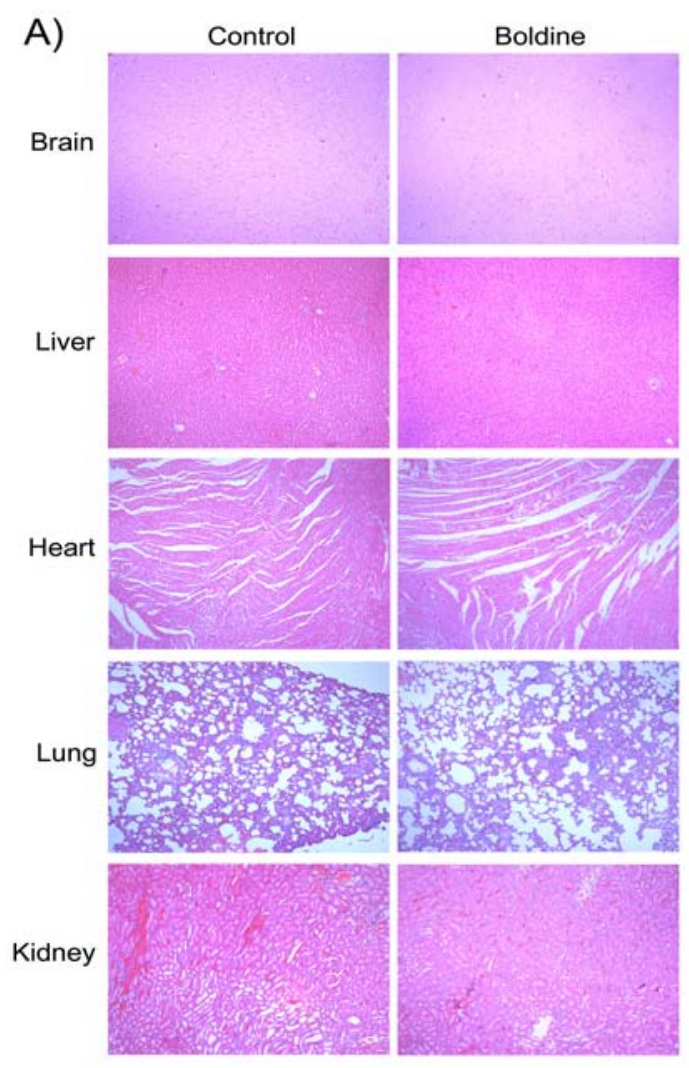

B)

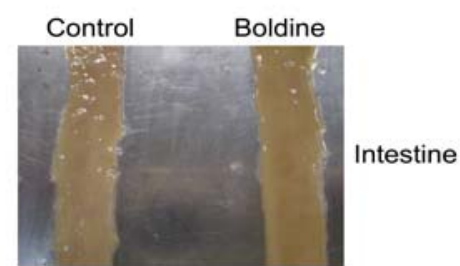

Figure 2: Toxicological evaluation in healthy animals. (A) Representative microphotographs showing H\&E-stained sections of brain, liver, lung, kidney and heart of control group and boldine-treated glioma-implanted rats; magnification 40x. (B) Representative intestine sections.

\begin{tabular}{|l|l|l|l|}
\hline & \multicolumn{1}{|c|}{ Control } & \multicolumn{1}{c|}{ Vehicle } & \multicolumn{1}{c|}{ Boldine } \\
\hline Lymphocytes & $81 \pm 1.7$ & $83.75 \pm 6.8$ & $76 \pm 9.6$ \\
\hline Neutrophils & $11 \pm 1.7$ & $6.75 \pm 5.1$ & $19.6 \pm 7.5$ \\
\hline Monocytes & $6,6 \pm 4,1$ & $8.5 \pm 6.4$ & $4 \pm 2.6$ \\
\hline Eosinophils & $1 \pm 1$ & $0.5 \pm 0.6$ & $0.3 \pm 0.5$ \\
\hline Basophils & $0.3 \pm 0.5$ & $0.5 \pm 0.6$ & 0 \\
\hline
\end{tabular}

Manual WBC differential (100 WBCs were found in a blood smear, counted, and categorized according to type) of healthy rats treated with boldine.

Table 1: Hematological Analysis.

showed C6 cells growing in the intracerebral, intraventricular and intraparenchymal spaces (data not shown). H\&E examination also shows that the untreated tumor presented nuclear pleomorfism, foci of tumor necrosis, intratumoral hemorrhage, lymphocytic infiltration and vascular proliferation, which are characteristics of glioblastoma multiforme in humans (Table 2). These characteristics were less pronounced when rats were treated with boldine indicating a less 


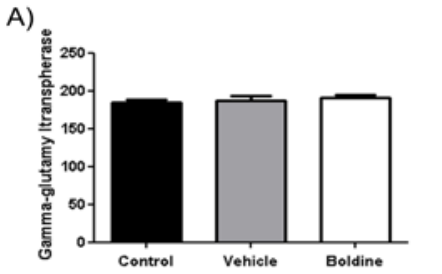

B)

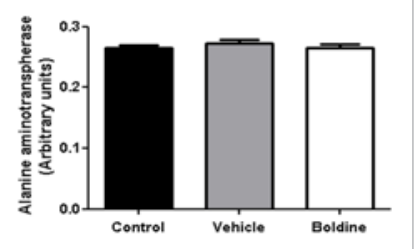

C)

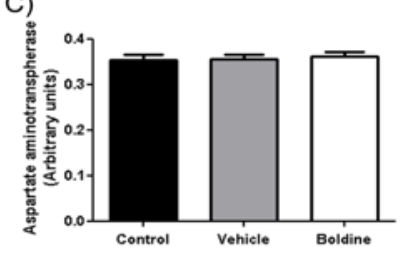

D)

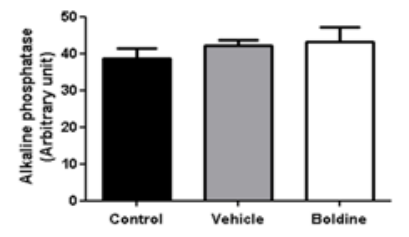

Figure 3: Enzymatic activity in healthy animals treated with boldine. Data show that boldine did not interfere in (A) Gamma-GT, (B) ALT, (C) AST and (D) alkaline phosphatase activities. The values are represented as means \pm S.D. of all animals used ( $n=4$ per group).

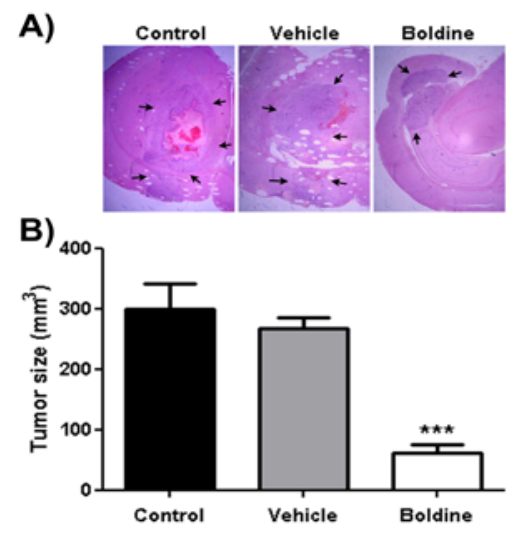

Figure 4: Boldine inhibits glioma growth in vivo. Animals were treated, as described in Section 2. Tumor size was measured 20 days after implantation of $\mathrm{C} 6$ cells by three hematoxylin and eosin (H\&E) sections of each tumor. For tumor size quantification, images were captured using a digital camera connected to a microscope and total volume $\left(\mathrm{mm}^{3}\right)$ was determined using Image Tool SoftwareTM. (A) Representative tumor images of the control, vehicle and boldine-treated groups, magnification 10x; (B) Tumor size quantification of implanted gliomas. The values are represented as means \pm S.D (Control group -6 animals; Vehicle group -6 animals; boldine-treated group -8 animals). Data were analyzed by ANOVA followed by Tukey's test. ${ }^{* * *} p<0.001$.

\begin{tabular}{|l|l|l|l|}
\hline & Control (n=6) & Vehicle (n=6) & \multicolumn{1}{|c|}{ Boldine (n=8) } \\
\hline $\begin{array}{l}\text { Coagulative } \\
\text { necrosis }\end{array}$ & $5 / 6(83,3 \%)$ & $6 / 6(100 \%)$ & $5 / 8(62,5 \%)$ \\
\hline $\begin{array}{l}\text { Intratumoral } \\
\text { hemorrhage }\end{array}$ & $6 / 6(100 \%)$ & $6 / 6(100 \%)$ & $6 / 8(75 \%)$ \\
\hline $\begin{array}{l}\text { Vascular } \\
\text { proliferation }\end{array}$ & $6 / 6(100 \%)$ & $6 / 6(100 \%)$ & $7 / 8(87,5 \%)$ \\
\hline Microabscess & $3 / 6(50 \%)$ & $4 / 6(66,6 \%)$ & $2 / 8(25 \%)$ \\
\hline $\begin{array}{l}\text { Lymphocytic } \\
\text { infiltration }\end{array}$ & $6 / 6(100 \%)$ & $6 / 6(100 \%)$ & $6 / 6(100 \%)$ \\
\hline $\begin{array}{l}\text { Peripheral } \\
\text { pseudopalisading }\end{array}$ & $3 / 6(50 \%)$ & $3 / 6(50 \%)$ & $5 / 8(62,5 \%)$ \\
\hline
\end{tabular}

The histological variables were regarded as present or absent. $(n=$ number of animals per group)

Table 2: Histological characteristics of implanted gliomas. invasive/proliferative tumor. Although the histological features were based on qualitative analysis of "presence or absence" of the alterations (percentage/proportion of animals), they clearly appear to be lower in boldine-treated rats when compared do control rats (Figure 5).

\section{Immunohistochemical analysis}

Immunohistochemical analysis with antibodies against the proliferation marker Ki67 revealed that cell proliferation was reduced by boldine treatment in rats implanted with $\mathrm{C} 6$ cells, indicating that these tumors are less proliferative and, consequently, less invasive than control tumors (Table 3) (Figure 6). Ki67 positive glioma cell nuclei were counted in at least 5 fields in high magnification (400x). Since tumor growth depends on the ability to induce angiogenesis, we also performed immunohistochemical experiments to determine a possible influence in Vascular Endothelial Growth Factor (VEGF) expression, a major angiogenic factor in glioma. Our results demonstrate no difference in VEGF staining between groups. Representative images are shown in Figure 7.

\section{Discussion}

Many of our present medicines are directly or indirectly derived from higher plants. Clinical plant-based research has made particularly

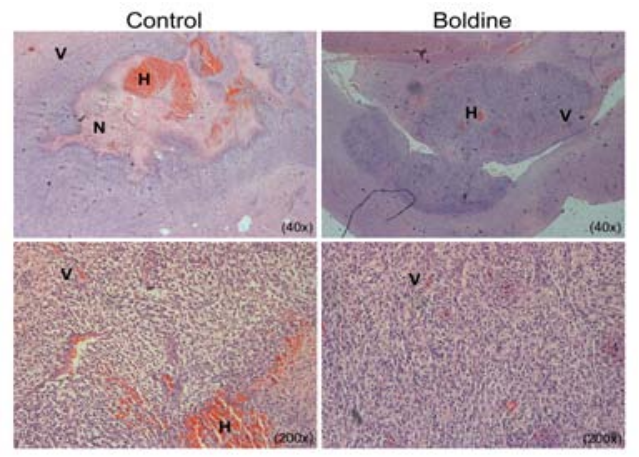

Figure 5: Histological analysis of implanted gliomas. The sections of implanted rat glioma were stained with hematoxylin and eosin (H\&E), as described in Section 2. Representative pictures of histological characteristics seen in rats of control group and treated group. Legends: $(\mathrm{N})$ necrosis, $(\mathrm{V})$ microvascular proliferation, hemorrhages $(\mathrm{H})$, Magnification 40x, 200x.

\begin{tabular}{|l|l|l|l|}
\hline & Control & Vehicle & Boldine \\
\hline Ki67 & $50 \pm 16$ & $63 \pm 23$ & $13 \pm 6,4^{*}$ \\
\hline
\end{tabular}

Ki67 results are expressing by percentage of positive cells. Data are the means \pm S.D. ANOVA followed by Tukey's test; * $p<0,05$

Table 3: Immunohistochemical analysis of Ki67 in implanted gliomas.

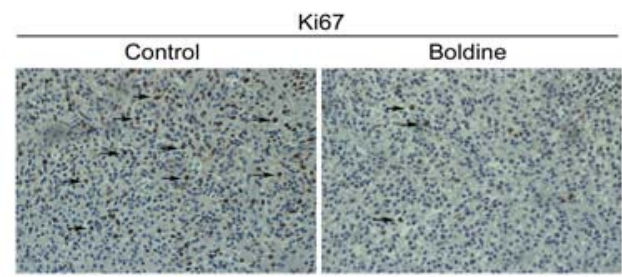

Figure 6: Immunohistochemical staining of Ki67 in implanted gliomas. Glioma cell proliferation was assessed by immunostaining for Ki67 positive glioma cell nuclei (arrows). Representative pictures of immunohistochemical analysis in control group and in boldine-treated group. Magnification 400x. 


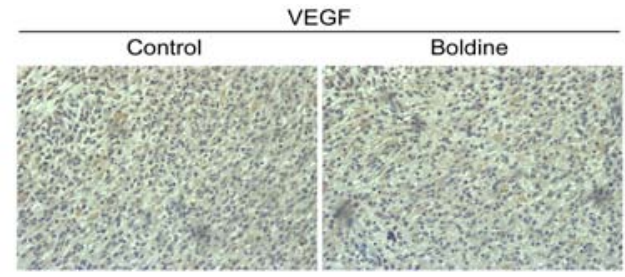

Figure 7: Immunohistochemical staining of VEGF in implanted gliomas. Glioma cell angiogenesis was assessed by VEGF staining. Representative pictures of immunohistochemical analysis in control group and in boldine-treated group. Magnification 400x.

rewarding progress in the important fields of anticancer therapy, such as curcumin [19,20], resveratrol [21], and others. In this study we propose a novel natural compound, the alkaloid boldine, for the development of a new anticancer agent, which could be used in conjunction with the established chemotherapies regimens. The precedent of the prolonged tradition of pharmaceutical use of boldine and boldine-containing boldo preparations suggesting that boldine exhibits low toxicity and the fact that this alkaloid is present in high concentrations in the bark of the Chilean boldo tree (Peumus boldus Mol., Monimiaceae), makes it an interesting candidate for development as a natural drug [2].

In the present study, we performed toxicological experiments in healthy Wistar rats after treatment with $50 \mathrm{mg} / \mathrm{Kg}$ of boldine administered intraperitoneally for 14 days. This treatment regimen did not cause mortality in animals. In fact, there are studies demonstrating that high doses are needed to induce death in several mammalian species (e.g., $15 \mathrm{~g}$ of boldine, administered orally, were necessary to kill a $12 \mathrm{~kg} \mathrm{dog}$ [2]). Studies by Kreitmair (1952) [3] reported that 500 and $1000 \mathrm{mg} / \mathrm{kg}$ (p.o.) were required to induce the death of mice and guinea pigs, respectively. Studies conducted later by L'evy-AppertCollin and L'evy (1977) [22], estimated a LD50 of $250 \mathrm{mg} / \mathrm{kg}$ (i.p.) in mice. The necropsy of the rats at the end of boldine treatment in our study did not show any macroscopic changes in the observed organs, and their weights did not differ from those of the control group (Figure 1B). Microscopic investigation of these organs by H\&E analysis demonstrated absence of toxicity (Figure 2A). Because boldine was demonstrated to be an inhibitor of prostaglandin biosynthesis [7], the occurrence of gastrointestinal damage was evaluated. No lesions were found in stomach or intestine after the treatment regimen employed.

To evaluate the possible liver damage in the boldine-treated healthy rats, we measured the levels of the enzymes gammaglutamyl transpherase, alanine aminotransaminase, aspartate aminotransaminase and alkaline phosphatase in the rat blood serum. None of the treated animals presented significant alterations in the investigated enzymes, discarding hepatic damage (Figure 3). Studying the effects of long-term administration of boldine, Almeida and colleagues (2000) [23], did also not observed hepatotoxicity, assessed by blood transaminases or urea levels, in rats given boldine p.o. daily at $500 \mathrm{mg} / \mathrm{kg}$ for 30 and 60 days, but a low degree of toxicity was seen at $800 \mathrm{mg} / \mathrm{kg}$.

Manual white blood cells differential was performed in blood smears stained with May-Grünwald-Giemsa. Analyzing the results, we found that the healthy animals treated with boldine had a tendency to increase the population of neutrophils. Earlier work by GonzálezCabello and colleagues (1994) [5] suggested that boldine present immunomodulating properties on natural killer cells and Philipov and colleagues (1998) [24] found that boldine inhibit the in vitro concanavalin A-induced proliferation of mouse splenocytes. Although these works suggest some possible immunomodulating properties of boldine, the actual potential of this aporphine to modify cellular immune functions require confirmation and further assessment.

Our findings, in conjunction with the overall results available on the toxicity of boldine in the literature, point to a relatively low toxicity of this compound. However, its actual innocuousness in humans still remains to be established in further investigations.

To determine whether the natural alkaloid boldine is able to avert brain tumor growth in an authentic microenvironment, we examined the effects of the compound in an orthotopic and immunocompetent rat model of glioma that mirrors the hallmarks of human gliomas [18]. This in vivo model is one of the most useful tools for a variety of studies, especially for investigations of glial tumor biology, as well as for experimental chemotherapy of brain malignancies [25]. We treated C6-implanted Wistar rats with $50 \mathrm{mg} / \mathrm{kg} /$ day boldine, ip, from the 10th to the 20th day after glioma implantation. Our experiments demonstrate that the treatment significantly diminished the growth of implanted gliomas in rats after twenty days of tumor induction (Figure 4). In addition, pathological analysis demonstrated that malignant characteristics appeared to be lower in boldine-treated rats (Table 2 and Figure 5). Among these, the reduction of the mitotic index was significant in treated rats, as observed by Ki67 immunostaining (Figure 6), indicating a tumor less proliferative. The vehicle-treated group did not present any significant difference from control group in any of the parameters analyzed; therefore we did not present the data from this group in all figures.

Angiogenesis is an extremely important process for sustained tumor growth. Thus, it constitutes an important point in the control of cancer progression and its inhibition may be a valuable new approach to cancer therapy. Studies have demonstrated that C6 cells secrete VEGF, a major angiogenic factor in glioma, which takes part of a critical signaling pathway for regulation of tumor angiogenesis [26,27]. In our study, we assessed VEGF expression by immunohistochemical staining. The results indicate no difference in VEGF staining between rat tumors treated with boldine and the control group (Figure 7). The absent of any difference in VEGF staining could be due to the dose used in this study. Maybe, it is possible that highest doses could interfere in VEGF secretion by glioma cells. Further studies are necessary to clarify this hypothesis.

Effective therapies for glioblastomas are scarce, and long term survival is a rarity [28]. The limited success of current treatment regimens is mainly caused by the ability of malignant glioma cells to diffusely infiltrate the surrounding healthy brain [29], a hallmark of glioblastoma that is recapitulated in the rat model of glioma [18]. Therefore, any new modality to replace or support current treatments for malignant gliomas would be helpful. In summary, this is the first report demonstrating therapeutic effects of boldine on glioma growth in immunocompetent mice. The effectiveness of boldine as an antitumor agent in our in vivo glioma model and its relatively safety observed in the experiments with healthy animals now justify the pursuit of studies aimed to explore its pharmacokinetics, major biotransformation pathways, its actual innocuousness in humans and further its actual therapeutic value in phase 1 clinical studies. As combination therapies are promising in glioblastoma, boldine might serve as an adjunct to established chemotherapeutic drugs and/or radiation therapy. 
Citation: Gerhardt D, Bertola G, Bernardi A, Simões Pires EN, Frozza RL, et al. (2013) Boldine Attenuates Cancer Cell Growth in an Experimental Model of Glioma In vivo. J Cancer Sci Ther 5: 194-199. doi:10.4172/1948-5956.1000206

\section{Acknowledgements}

This work was supported by Conselho Nacional de Desenvolvimento Científico e Tecnológico (CNPq), Coordenação de Aperfeiçoamento de Pessoal de Níve Superior (Capes), Fundação de Amparo à Pesquisa do Estado do Rio Grande do Sul (FAPERGS) and FIPE-HCPA (Protocol 08-580).

\section{References}

1. Lanhers MC, Joyeux M, Soulimani R, Fleurentin J, Sayag M, et al. (1991) Hepatoprotective and anti-inflammatory effects of a traditional medicinal plant of Chile, Peumus boldus. Planta Med 57: 110-115.

2. Speisky H, Cassels BK (1994) Boldo and boldine: an emerging case of natura drug development. Pharmacol Res 29: 1-12.

3. Kreitmair H (1952) [Pharmacological effects of the alkaloid of Peumus boldus molina]. Pharmazie 7: 507-511.

4. Genest K, Hughes DW (1968) Natural products in Canadian pharmaceuticals II. Peumus boldus. Can J Pharm Sci 3:84-90.

5. González-Cabello R, Speisky H, Bannach R, Valenzuela A, Fehér J, et al. (1994) Effects of boldine on cellular immune functions in vitro. J Investig Allergol Clin Immunol 4: 139-145.

6. Speisky H, Squella JA, Núñez-Vergara LJ (1991) Activity of boldine on rat ileum. Planta Med 57: 519-522.

7. Backhouse N, Delporte C, Givernau M, Cassels BK, Valenzuela A, et al. (1994) Anti-inflammatory and antipyretic effects of boldine. Agents Actions 42: 114117.

8. Gotteland M, Jimenez I, Brunser O, Guzman L, Romero S, et al. (1997) Protective effect of boldine in experimental colitis. Planta Med 63: 311-315

9. Zetler G (1988) Neuroleptic-like, anticonvulsant and antinociceptive effects of aporphine alkaloids: bulbocapnine, corytuberine, boldine and glaucine. Arch Int Pharmacodyn Ther 296: 255-281.

10. Speisky H, Cassels BK, Lissi EA, Videla LA (1991) Antioxidant properties of the alkaloid boldine in systems undergoing lipid peroxidation and enzyme inactivation. Biochem Pharmacol 41: 1575-1581.

11. Cederbaum Al, KukieÅ,ka E, Speisky H (1992) Inhibition of rat liver microsomal lipid peroxidation by boldine. Biochem Pharmacol 44: 1765-1772.

12. Bannach R, Valenzuela A, Cassels BK, Nunez-Vergara LJ, Speisky H (1996) Cytoprotective and antioxidant effects of boldine on tert-butyl hydroperoxideinduced damage to isolated hepatocytes. Cell Biol Toxicol 12: 89-100.

13. Martínez LA, Ríos JL, Payá M, Alcaraz MJ (1992) Inhibition of nonenzymic lipid peroxidation by benzylisoquinoline alkaloids. Free Radic Biol Med 12: 287-292.

14. Gerhardt D, Horn AP, Gaelzer MM, Frozza RL, Delgado-Cañedo A, et al (2009) Boldine: a potential new antiproliferative drug against glioma cell lines. Invest New Drugs 27: 517-525.
15. Gerhardt D, Bertola G, Dietrich F, Figueiró F, Zanotto-Filho, et al. (2012) Boldine induces cell cycle arrest and apoptosis in T24 human bladder cancer cell line via regulation of ERK, AKT AND GSK-3ß. Urol Onol, (In Press).

16. Dehdashti AR, Hegi ME, Regli L, Pica A, Stupp R (2006) New trends in the medical management of glioblastoma multiforme: the role of temozolomide chemotherapy. Neurosurg Focus 20: E6.

17. Takano T, Lin JH, Arcuino G, Gao Q, Yang J, et al. (2001) Glutamate release promotes growth of malignant gliomas. Nat Med 7: 1010-1015.

18. Morrone FB, Oliveira DL, Gamermann P, Stella J, Wofchuk S, et al. (2006) In vivo glioblastoma growth is reduced by apyrase activity in a rat glioma model. BMC Cancer 6: 226 .

19. Guo LD, Chen XJ, Hu YH, Yu ZJ, Wang D, et al. (2013) Curcumin inhibits proliferation and induces apoptosis of human colorectal cancer cells by activating the mitochondria apoptotic pathway. Phytother Res 27: 422-430.

20. Zanotto-Filho A, Braganhol E, Edelweiss MI, Behr GA, Zanin R, et al. (2012) The curry spice curcumin selectively inhibits cancer cells growth in vitro and in preclinical model of glioblastoma. J Nutr Biochem 23: 591-601.

21. Zamin LL, Filippi-Chiela EC, Dillenburg-Pilla P, Horn F, Salbego C, et al. (2009) Resveratrol and quercetin cooperate to induce senescence-like growth arrest in C6 rat glioma cells. Cancer Sci 100: 1655-1662.

22. Lévy-Appert-Collin MC, Lévy J (1977) [Galenic preparations from Peumus boldus leave (Monimiacea) (author's transl)]. J Pharm Belg 32: 13-22.

23. Almeida ER, Melo AM, Xavier H (2000) Toxicological evaluation of the hydro-alcohol extract of the dry leaves of Peumus boldus and boldine in rats. Phytother Res 14: 99-102.

24. Philipov S, Ivanovska N, Nikolova P (1998) Glaucine analogues as inhibitors of mouse splenocyte activity. Pharmazie 53: 694-698.

25. Barth RF, Kaur B (2009) Rat brain tumor models in experimental neurooncology: the C6, 9L, T9, RG2, F98, BT4C, RT-2 and CNS-1 gliomas. J Neurooncol 94: 299-312.

26. Lamszus K, Brockmann MA, Eckerich C, Bohlen P, May C et al. (2005) Inhibition of glioblastoma angiogenesis and invasion by combined treatments directed against vascular endothelial growth factor receptor-2, epidermal growth factor receptor, and vascular endothelial-cadherin. Clin Cancer Res 11 : 4934-4940.

27. Plate KH, Breier G, Millauer B, Ullrich A, Risau W (1993) Up-regulation of vascular endothelial growth factor and its cognate receptors in a rat glioma model of tumor angiogenesis. Cancer Res 53: 5822-5827.

28. Bähr O, Herrlinger U, Weller M, Steinbach JP (2009) Very late relapses in glioblastoma long-term survivors. J Neurol 256: 1756-1758.

29. Claes A, Idema AJ, Wesseling $P$ (2007) Diffuse glioma growth: a guerilla war Acta Neuropathol 114: 443-458. 